\title{
Sebaceous carcinoma arising from sebaceoma
}

\author{
Da Woon Lee', \\ Si Hyun Kwak ${ }^{1}$, \\ Jun Hyuk Kim ${ }^{1}$, \\ Je Yeon Byeon ${ }^{1}$, \\ Hyun Joo Lee', \\ Hwan Jun Choi ${ }^{1}$ \\ Departments of ${ }^{1}$ Plastic and \\ Reconstructive Surgery and ${ }^{2}$ Pathology, \\ Soonchunhyang University College of \\ Medicine, Cheonan, Korea
}

\begin{abstract}
Sebaceous neoplasms are rare adnexal tumors that can present a challenge to clinicians. Only four cases of sebaceous carcinoma with sebaceoma have been reported in the literature. Herein, we describe the case of a sebaceous carcinoma originating from a sebaceoma in a solitary nodule of the posterior neck. Immunohistochemically, the tumor cells were strongly positive for epithelial membrane antigen and p53. It is possible that adnexal carcinomas may arise from malignant transformation of their benign counterparts as well as de novo. Malignant transformation was likely in this case because the lesion was composed of distinct benign and malignant components, and the benign component showed the typical histopathological features of sebaceoma. This case underscores the fact that partial and superficial biopsies sometimes may not provide the correct diagnosis. If a surgeon suspects malignancy based on a clinical examination, then it is mandatory to perform a deep biopsy.
\end{abstract}

Keywords: Adnexal and skin appendage neoplasms / Benign neoplasms / Carcinoma, skin appendage

\section{INTRODUCTION}

Sebaceous neoplasms are rare, clinically challenging adnexal tumors. Due to their rarity and the wide spectrum of skin lesions, confusion exists regarding their nomenclature, associated syndromes, and treatment.

Sebaceoma is a distinctive benign neoplasm of the adnexal epithelium with differentiation toward sebaceous structures; it presents as a circumscribed nodular lesion that is rarely diagnosed before a histological examination [1]. Sebaceoma is often misdiagnosed as sebaceous adenoma, which is more organized and usually has a less prominent basaloid component. Sebaceous carcinoma is an extremely rare cutaneous tumor with an estimated incidence of approximately 1-2 per 1,000,000 per

\section{Correspondence: Hwan Jun Choi}

Department of Plastic and Reconstructive Surgery, Soonchunhyang University Cheonan Hospital, Soonchunhyang University College of Medicine,

31 Suncheonhyang 6-gil, Dongnam-gu, Cheonan 31151, Korea

E-mail: iprskorea@gmail.com

This work was supported by the National Research Foundation of Korea (NRF) grant funded by the Korea government (MSIT) (2020R1A2C1100891), and was supported Soonchunhyang University Research Fund.

Received February 17, 2021 / Revised April 8, 2021 / Accepted April 20, 2021 year. Sebaceous carcinoma usually develops in the periocular area, but it also occurs elsewhere in the head and neck and is usually accompanied by rapid growth, tenderness, and ulceration [2].

Sebaceous carcinoma arising from sebaceoma is an extremely rare condition. Four cases were reported by Kazakov et al. [3], who did not establish a standardized size of the free margin. Instead, those cases were resected with an empirical margin, as presented in Table 1. This condition usually occurs in the craniofacial area. The mean age of onset is 49 years, and there is no sex predilection. We report an extremely rare case of sebaceous carcinoma occurring within a sebaceoma and review the relevant literature.

\section{CASE REPORT}

A 74-year-old Asian woman presented with a solitary nodular skin lesion on the posterior neck that had developed 1 year previously. She had already been diagnosed with sebaceoma with a small portion of seborrheic keratosis 1 month ago by a partial excisional biopsy. The physical examination revealed a firm, 
Table 1. Sebaceous gland carcinoma originating from sebaceous lesions published in the only report to date [3]

\begin{tabular}{llclll}
\hline Case & Sex & Age $(\mathrm{yr})$ & Location & Clinical impression & Treatment \\
\hline 1 & Male & 43 & Right forearm & $2 \times 1.5 \mathrm{~cm}$ solitary nodule & Excision \\
2 & Male & 44 & Scalp & $1 \mathrm{~cm}$ solitary nodule & Excision \\
3 & Male & 59 & Scalp & $3 \times 2 \mathrm{~cm}$ solitary nodule & Excision \\
4 & Female & 49 & Occipital area & $2.5 \mathrm{~cm}$ solitary nodule & Excision \\
\hline
\end{tabular}

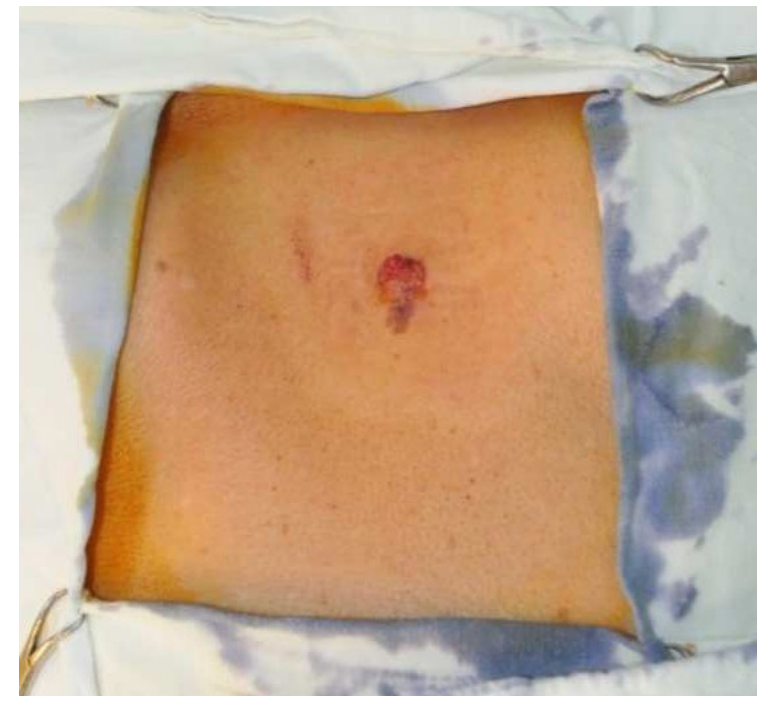

Fig. 1. Preoperative photograph. A firm, brown-to-reddish skin nodule with discoloration was observed on the posterior neck.

brown-to-reddish skin nodule about $10 \mathrm{~mm}$ in diameter with an erosive dimple on the upper portion (Fig. 1). There was no other abnormality of other organs and systems. The patient also had no significant past medical history. Under local anesthesia, the nodule was completely excised with a 5-mm free margin.

Histological examination of the biopsy taken from the lesion revealed a sebaceous carcinoma arising in a sebaceoma. The large nodule in the upper part of the lesion corresponded to sebaceous carcinoma with ulceration. The specimen consisted of multiple variably-sized, discrete nodules and was centered in the superficial dermis (Fig. 2). The sebaceoma was composed of an admixture of basaloid germinative sebaceous cells and mature sebocytes. There was no nuclear pleomorphism and the mitotic activity was sparse. The sebocytes appeared mature with clear to lightly eosinophilic bubbly cytoplasm. The sebaceous carcinoma displayed an infiltrating growth pattern. The tumor cells showed marked nuclear pleomorphism and frequent mitoses. In addition, the sebaceous carcinoma was immunohistochemically characterized by strong positivity for epithelial membrane antigen. Mutations and nuclear accumulation of p53 tumor suppressor gene expression were also noted as features that may be correlated with a poor outcome. The pa- tient was discharged without any postoperative complications. There was no sign of recurrence or metastasis after excision during a 5-month follow-up period.

\section{DISCUSSION}

This case is interesting because the lesion was composed of distinct benign and malignant components, and the benign component showed the typical histopathological features of sebaceoma. Few cases of sebaceous carcinoma arising in a preexisting sebaceoma have been reported in the literature. Although sebaceous carcinoma is sometimes accompanied by sebaceoma, these two types of neoplasms (sebaceous carcinoma and sebaceoma) are usually sharply segregated [4]. Adnexal carcinomas, such as clear cell hidradenocarcinoma and microcystic adnexal carcinoma are generally thought to develop de novo $[5,6]$. However, some adnexal carcinomas arise from preexisting benign counterparts; for instance, $20 \%$ to $50 \%$ of porocarcinomas are associated with poromas. Most spiradenocarcinomas and cylindrocarcinomas arise from their benign counterparts. This evidence suggests that some adnexal carcinomas arise from malignant transformation of their benign counterparts as well as de novo $[7,8]$.

Sebaceoma, a term proposed by Troy and Ackerman [9], is a benign skin adnexal tumor with sebaceous differentiation, which can occur as a primary lesion or as a secondary tumor to either nevus sebaceous or seborrheic keratosis. The architectural pattern is characterized by sharp circumscription, smooth borders, and symmetry. The lesions have aggregations of basaloid undifferentiated sebocytes admixed with single or small clusters of mature vacuolated sebocytes, often in association with sebaceous duct-like structures and dense eosinophilic sclerosis. Like sebaceous adenomas, these neoplasms can be associated with Muir-Torre syndrome [10]. The major difference between sebaceoma and sebaceous adenoma is that the former has randomly scattered sebaceous cells without nuclear atypia, whereas the latter displays more highly organized, irregularly shaped lobules of sebaceous cells with an outer rim of more than a single layer of small germinal cells. There are no known immunohistochemical differences in addition to the architec- 

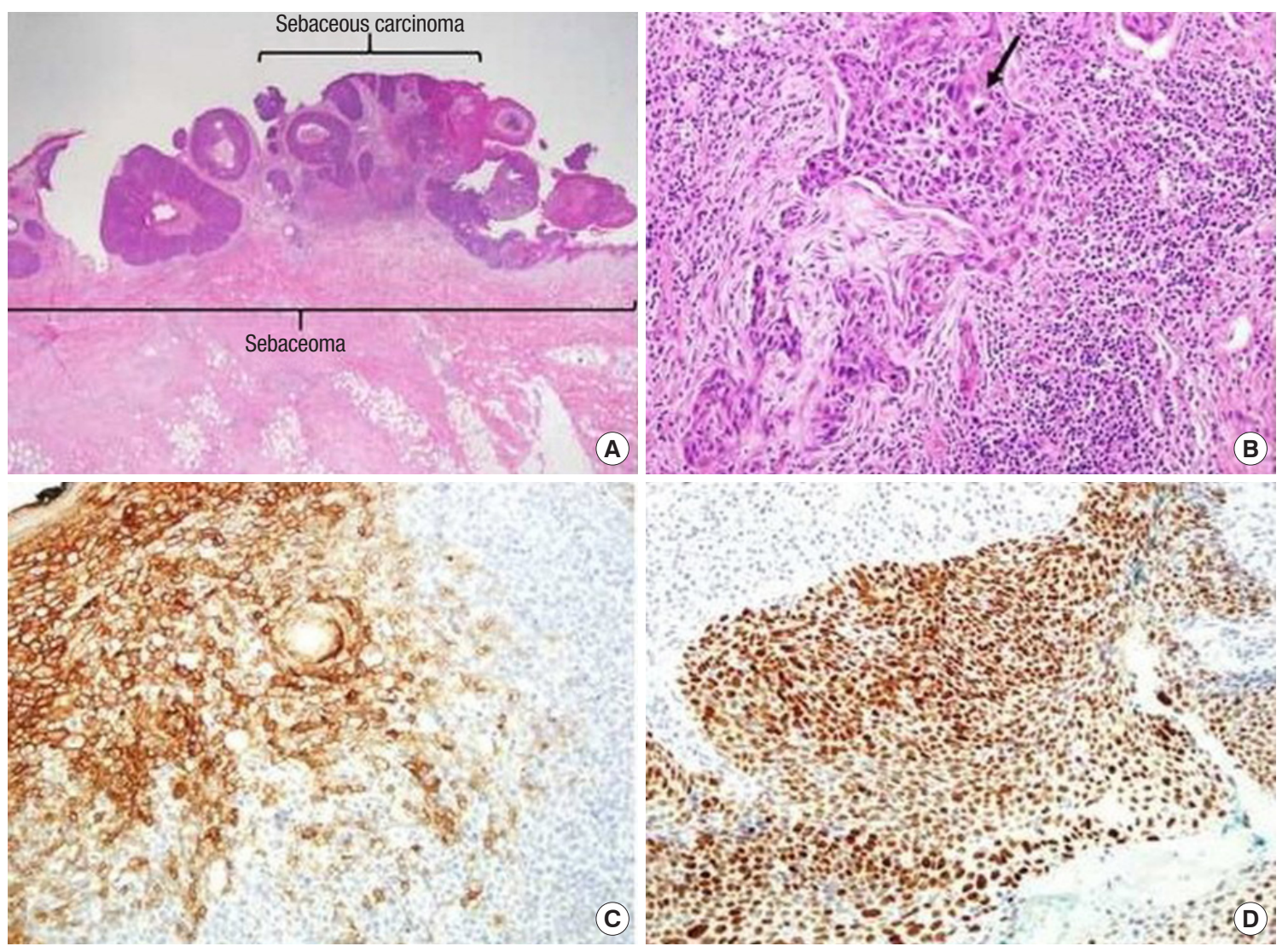

Fig. 2. Histologic findings. (A) Sebaceous carcinoma arising in sebaceoma. The tumor consists of multiple variably-sized, discrete nodules and is centered in the superficial dermis $(\mathrm{H} \& \mathrm{E}, \times 1.25)$. (B) The sebaceous carcinoma displays an infiltrating growth pattern (arrow) (H\&E, $\times 20$ ). (C) Sebaceous carcinoma is characterized by strong epithelial membrane antigen positivity (immunohistochemistry stain, $\times 20$ ). (D) Mutations and nuclear accumulation of p53 tumor suppressor gene expression are also noted (immunohistochemistry stain, $\times 20$ ).

tural ones. Clinically, the lesions are described as yellowish papules with rolled borders, usually on the scalp, face, and neck. They are typically solitary and have a diameter of $1 \mathrm{~cm}$, but larger lesions have been reported. Ulceration and bleeding are common findings. As with sebaceous adenoma, excision is considered an adequate treatment for these lesions [11]. Kantrow et al. [5] and Nakai et al. [6] reported patients presenting with sebaceoma and a combined nevus sebaceous.

Sebaceous carcinoma is a rare neoplasm, of which $75 \%$ of cases occur on the periorbital region and approximately $25 \%$ occur in the extraocular region. Of those cases, $70 \%$ arise in the head and neck region, with the parotid gland being the most common location. The most frequent clinical presentation of sebaceous carcinoma is a painless subcutaneous nodule. Other presentations include diffuse thickening of the skin, pedunculated lesions, or an irregular mass. Its clinical manifestations are often mistaken for benign conditions, resulting in a delayed diagnosis and management [12]. Metastasis to regional lymph nodes and other sites is common in these situations. Pathologists must be aware of the varied clinical manifestations of this condition, its histopathological morphology, and its multiple biomarkers to ensure an accurate diagnosis $[13,14]$. The protean appearance of sebaceous carcinoma complicates the diagnosis. It often presents with an appearance similar to more common benign lesions and is frequently misdiagnosed. It is not unusual for a sebaceous carcinoma to be treated multiple times as a chalazion before the diagnosis is made. An incomplete biopsy specimen, either because of its proximity to the eye or because sebaceous carcinoma is not in the clinical differential diagnosis at the time, can both complicate matters and delay therapy. Treatment is surgical, with Mohs micrographic surgery having the best results; there is an $11 \%$ recurrence rate after Mohs micrographic surgery and a 30\% rate after standard excision [15].

Sebaceous carcinoma arises from two possible cell types: pluripotent cells with the capacity for sebaceous differentiation or ectopic sebaceous cells that develop during embryogenesis. Other reported locations include the submandibular glands, extremities, toes, penis, chest wall, sole of the foot, external auditory canal, and anterior neck region. The varied origin of this neoplasm highlights the importance of considering sebaceous 
carcinoma regardless of the anatomical location [16]. Several papers have been published on the relationship between sebaceous cancer and human papillomavirus $[17,18]$. Overexpression of p53 is involved in the carcinogenesis of this tumor, and is a risk factor suggesting a poor prognosis [17]. The histogenesis of sebaceous carcinomas remains unclear. Intraepidermal or intraepithelial sebaceous carcinoma in the epidermis or conjunctiva, as well as sebaceous carcinoma in association with a Bowen's disease-like lesion, may suggest de novo histogenesis of sebaceous carcinoma from abnormal sebaceous germinative cells within the epidermis or conjunctival epithelium [19].

In conclusion, partial and superficial biopsies may not provide the correct diagnosis in many cases. The need for deep biopsy in cases of skin tumors should be emphasized. In fact, if a surgeon suspects malignancy on clinical examination, then it is mandatory to perform a deep biopsy. Time is important in such cases because sebaceous carcinoma is an aggressive tumor that spreads to deeper structures and also metastasizes to other sites very rapidly. Pathological examinations are limited regarding microscopic features in certain cases of very small and superficial biopsies that do not show a clear-cut malignant tumor. Therefore, the authors emphasize the importance of awareness of the possibility of sebaceous carcinoma with combined sebaceoma, which may lead to a better evaluation and treatment of this condition.

\section{NOTES}

\section{Conflict of interest}

No potential conflict of interest relevant to this article was reported.

\section{Ethical approval}

The study was approved by the Institutional Review Board of Soonchunhyang University Cheonan Hospital (IRB No. 202102-051) and performed in accordance with the principles of the Declaration of Helsinki. Written informed consent was obtained.

\section{Patient consent}

The patient provided written informed consent for the publication and the use of her images.

\section{ORCID}

Da Woon Lee https://orcid.org/0000-0002-6969-5643 Si Hyun Kwak Jun Hyuk Kim Je Yeon Byeon https://orcid.org/0000-0001-9594-700X https://orcid.org/0000-0002-0266-8480 https://orcid.org/0000-0002-1948-0687
Hyun Joo Lee https://orcid.org/0000-0002-0335-5996

Hwan Jun Choi

\section{Author contribution}

Conceptualization: DWL, HJC, JYB. Data curation: SHK, JYB, HJL. Formal analysis: DWL, SHK, JYB. Funding acquisition: JYB. Methodology: JYB, HJL. Project administration: DWL, HJC, JYB. Visualization: DWL, HJC, JYB. Investigation: DWL. Software: HJC. Supervision: HJC, JHK. Validation: HJC, JHK. Writing - original draft: DWL, JYB. Writing - review \& editing: DWL.

\section{REFERENCES}

1. Iacobelli J, Harvey NT, Wood BA. Sebaceous lesions of the skin. Pathology 2017;49:688-97.

2. Lazar AJF, McKee PH. Tumors and related lesions of the sebaceous glands. In: McKee PH, Calonje E, Granter SR, editors. Pathology of the skin: with clinical correlation. 4th ed. Philadelphia: Mosby Elsevier; 2012. p. 1495-6.

3. Kazakov DV, Kutzner H, Rutten A, Mukensnabl P, Michal M. Carcinoid-like pattern in sebaceous neoplasms: another distinctive, previously unrecognized pattern in extraocular sebaceous carcinoma and sebaceoma. Am J Dermatopathol 2005; 27:195-203.

4. Kazakov DV, Calonje E, Zelger B, Luzar B, Belousova IE, Mukensnabl P, et al. Sebaceous carcinoma arising in nevus sebaceus of Jadassohn: a clinicopathological study of five cases. Am J Dermatopathol 2007;29:242-8.

5. Kantrow SM, Ivan D, Williams MD, Prieto VG, Lazar AJ. Metastasizing adenocarcinoma and multiple neoplastic proliferations arising in a nevus sebaceus. Am J Dermatopathol 2007; 29:462-6.

6. Nakai K, Yoneda K, Moriue J, Moriue T, Matsuoka Y, Kubota Y. Sebaceoma, trichoblastoma and syringocystadenoma papilliferum arising within a nevus sebaceous. J Dermatol 2008;35: 365-7.

7. Robson A, Greene J, Ansari N, Kim B, Seed PT, McKee PH, et al. Eccrine porocarcinoma (malignant eccrine poroma): a clinicopathologic study of 69 cases. Am J Surg Pathol 2001;25:71020.

8. Granter SR, Seeger K, Calonje E, Busam K, McKee PH. Malignant eccrine spiradenoma (spiradenocarcinoma): a clinicopathologic study of 12 cases. Am J Dermatopathol 2000;22:97103.

9. Troy JL, Ackerman AB. Sebaceoma: a distinctive benign neoplasm of adnexal epithelium differentiating toward sebaceous cells. Am J Dermatopathol 1984;6:7-13. 
10. Shalin SC, Lyle S, Calonje E, Lazar AJ. Sebaceous neoplasia and the Muir-Torre syndrome: important connections with clinical implications. Histopathology 2010;56:133-47.

11. Eisen DB, Michael DJ. Sebaceous lesions and their associated syndromes: part I. J Am Acad Dermatol 2009;61:549-60.

12. Toto V, Colapietra A, Alessandri-Bonetti M, Vincenzi B, Devirgiliis $\mathrm{V}$, Panasiti $\mathrm{V}$, et al. Upper eyelid Merkel cell carcinoma treated with neoadjuvant chemotherapy and surgical excision. Arch Craniofac Surg 2019;20:121-5.

13. Kazakov DV, Kutzner H, Spagnolo DV, Rutten A, Mukensnabl P, Michal M. What is extraocular cutaneous sebaceous carcinoma in situ? Am J Dermatopathol 2010;32:857-8.

14. Boniuk M, Zimmerman LE. Sebaceous carcinoma of the eyelid, eyebrow, caruncle and orbit. Int Ophthalmol Clin 1972;12: 225-57.

15. Ansai S, Arase S, Kawana S, Kimura T. Immunohistochemical findings of sebaceous carcinoma and sebaceoma: retrieval of cytokeratin expression by a panel of anti-cytokeratin monoclonal antibodies. J Dermatol 2011;38:951-8.

16. Misago N, Narisawa Y. Ripple/carcinoid pattern sebaceoma with apocrine differentiation. Am J Dermatopathol 2011;33: 94-7.

17. Hayashi N, Furihata M, Ohtsuki Y, Ueno H. Search for accumulation of $\mathrm{p} 53$ protein and detection of human papillomavirus genomes in sebaceous gland carcinoma of the eyelid. Virchows Arch 1994;424:503-9.

18. Stagner AM, Afrogheh AH, Jakobiec FA, Iacob CE, Grossniklaus HE, Deshpande V, et al. p16 expression is not a surrogate marker for high-risk human papillomavirus infection in periocular sebaceous carcinoma. Am J Ophthalmol 2016;170: 168-75.

19. Izumi M, Mukai K, Nagai T, Matsubayashi J, Iwaya K, Chiu CS, et al. Sebaceous carcinoma of the eyelids: thirty cases from Japan. Pathol Int 2008;58:483-8. 